\title{
STATISTICAL ANALYSIS OF INFLOW AND STRUCTURAL RESPONSE DATA FROM THE LIST PROGRAM ${ }^{* \dagger}$
}

\author{
Luke D. Nelson ${ }^{1}$ \\ Lance Manuel ${ }^{1}$ \\ Herbert J. Sutherland ${ }^{2}$ \\ Paul S. Veers ${ }^{2}$ \\ ${ }^{1}$ Department of Civil Engineering, University of Texas at Austin, Austin, TX 78712 \\ ${ }^{2}$ Wind Energy Technology Department, Sandia National Laboratories, Albuquerque, NM 87185
}

\begin{abstract}
The Long-Term Inflow and Structural Test (LIST) program is gathering inflow and structural response data on a modified version of the Micon 65/13 wind turbine at a test site near Bushland, Texas. Data from 491 ten-minute time data records are analyzed here to determine the dependency of fatigue and extreme loads on inflow parameters.
\end{abstract}

Flap and edge bending moment ranges at a blade root are chosen as the structural response variable, $z$. Various parameters related to the inflow (including, for example, primary parameters, the mean and standard deviation of the hub-height horizontal wind speed, and secondary parameters, Reynolds stresses, vertical shear exponent, etc.) are each considered in an inflow parameter vector, $\boldsymbol{x}$. Time series for the structural response, $z$, are processed in order to obtain a structural response parameter, $y$, where in separate statistical studies, $y$ is taken to be either an equivalent fatigue load or an extreme load. This paper first describes a procedure by which the important "dependencies" of $y$ on the various variables contained in the inflow parameter vector, $\boldsymbol{x}$, may be determined considering all the available data. These dependencies of $y$ on $\boldsymbol{x}$ are then recomputed using only the data with above-rated mean wind speeds (taken to be approximately $13 \mathrm{~m} / \mathrm{s}$ ).

The procedure employed is similar to other previous studies, but we do not bin the data sets by wind speed since dependencies in one wind speed bin may be different from those in other bins. Also, our procedure, in sharp contrast to previous studies, examines each inflow parameter in the vector, $\boldsymbol{x}$, in a sequential analysis, rather than by using multivariate regression.

\footnotetext{
"Sandia is a multiprogram laboratory operated by Sandia Corporation, a Lockheed Martin company, for the U.S. Department of Energy under contract DE-AC04-94AL85000.

${ }^{*}$ This paper is declared a work of the U.S. Government and is not subject to copyright protection in the United States.
}

\section{INTRODUCTION}

Fundamental to the study of site-specific design loads for wind turbines is an understanding of the influence of inflow parameters on such loads. Inflow parameters might include, for example, mean wind speed, turbulence intensity, roughness length, transverse turbulence, and turbulence length scales for each wind component, to name a few.

The Long-Term Inflow and Structural Test (LIST) program managed by Sandia National Laboratories has made available continuous time series of atmospheric inflow conditions and structural response data from a wind turbine in Bushland, Texas (see Sutherland [1] and Berg and Zayas [2]). In previous studies, Sutherland $[3,4]$ employed the LIST data to assess the influence of atmospheric conditions on damageequivalent fatigue loads [5]. This same data set is employed in the present study. This study determines the dependencies that exist in blade root flap and edge bending moment fatigue and extreme loads on various inflow parameters.

In contrast with past analyses of these data, this study seeks to examine the ability of inflow parameters to explain the response behavior of a turbine sequentially (i.e., considering one inflow parameter at a time), rather than with a multivariate approach. Since there is a well-accepted practice of using the mean and standard deviation of the horizontal wind speed at the hub height to characterize the design environment, the dependence of these two parameters is first extracted from the loads data. Then, the next most influential parameter is identified and extracted. The goal is that such a procedure can provide a measure of which parameters are most likely to add understanding of the site influence on turbine loads in addition to the ones already in use. Also, the need, as in a multivariate regression, for the inflow parameters (independent variables) to be statistically independent is removed. 


\section{THE LIST DATA SET}

The LIST turbine is a three-bladed Micon $65 / 13 \mathrm{M}$ wind turbine that is being tested at a USDA site located near Bushland, Texas. The site is representative of most Great Plains commercial sites. For a complete description of the turbine, its instrumentation, and the site, see Sutherland [1,2]. A short description of the turbine and the measurement campaign is included here.

\section{The LIST Turbine}

The Micon 65/13M LIST turbine, see Fig. 1, is a fixedpitch turbine with a 3-phase $480 \mathrm{~V}$ asynchronous generator rated at $115 \mathrm{~kW}$. The generator operates at $1200 \mathrm{rpm}$ while the blades turn at a fixed $55 \mathrm{rpm}$. (Note that the standard Micon 65/13 turbine rotates at a fixed $45 \mathrm{rpm}$ ). The turbine is fitted with Phoenix $8 \mathrm{~m}$ blades that are based on Solar Energy Research Institute (SERI) airfoils. These "SERI" blades are $7.9 \mathrm{~m} \mathrm{(312}$ in) long and are equipped with tip brakes.

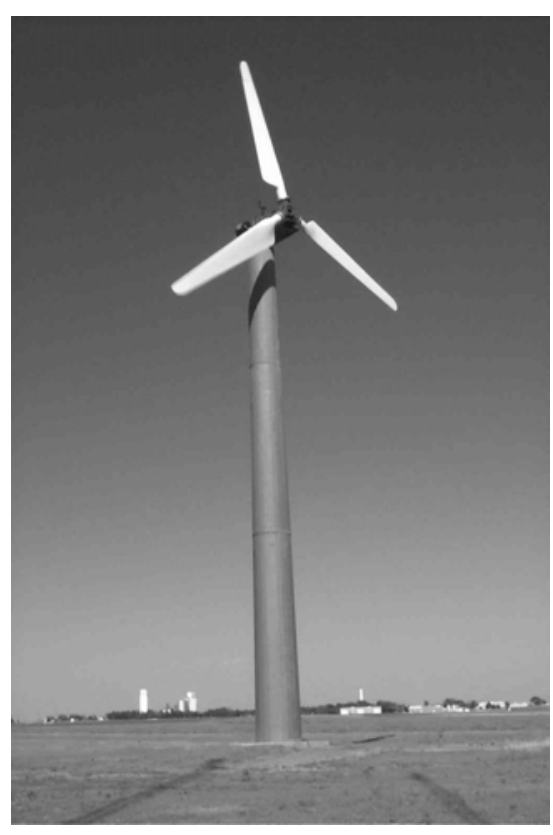

\section{Figure 1. The LIST Turbine near Bushland, Texas.}

\section{$\underline{\text { Data }}$}

From the LIST program, data are recorded and stored as ten-minute segments. The experiment is being monitored using a total of 60 sensors: 19 sensors measure structural response and 34 sensors measure

\footnotetext{
${ }^{\ddagger}$ SERI is now the National Renewable Energy Laboratory (NREL)
}

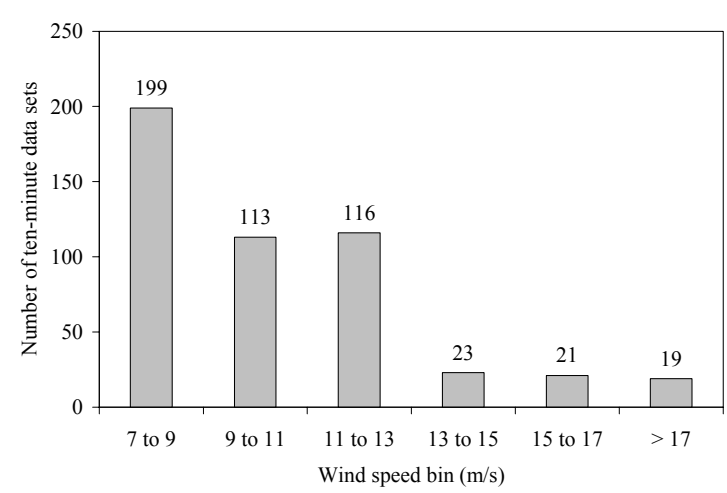

Figure 2. Distribution of 10-Minute Records Classified by Mean Wind Speed.

atmospheric conditions. A total of 1998 ten-minute records are available in this data set. This analysis uses a subset of the data, the records with mean wind speeds greater than $7 \mathrm{~m} / \mathrm{s}$ (comprising 491 ten-minute records). Figure 2 shows a distribution of the records used, classified according to mean horizontal wind speed. Of these records, 63 have mean wind speeds above the rated wind speed of the turbine (taken to be $13 \mathrm{~m} / \mathrm{s}$ here).

\section{NUMERICAL ANALYSIS}

Several authors have examined the influence of inflow parameters on fatigue loads. Fragoulis [6], Glinou and Fragoulis [7], and Sutherland [1,3] have examined the influence of various inflow parameters on equivalent fatigue loads. Kelley [8] has examined the influence of several parameters on the shape of the fatigue spectrum. The present study is one more study aimed at determining the influence of inflow parameters on both equivalent fatigue loads as well as extreme loads. Data from the LIST program are employed in this study.

\section{Structural Response}

The structural response, $z$, is taken directly from the available ten-minute time series records. In this study, this variable, $z$, will represent the edge or the flap bending moment at the root of a blade. The time series representing $z$ are processed to yield the variable, $y$, which is chosen to be, in separate statistical analyses, first, the damage-equivalent fatigue root bending moments (Sutherland $[3,4]$ ), and next, the maximum or extreme bending moment. The damage-equivalent load is the constant-amplitude load at a specified number of cycles that produces equivalent damage (in this case, 1000 cycles for each 10-minute sample, Sutherland [5]). The extreme load is the largest load amplitude in each 10-minute sample. The purpose of the proposed 
analysis is to determine the influence of the inflow parameters on damage-equivalent fatigue loads as well as extreme loads.

\section{Inflow Parameters}

The atmospheric time series data are processed to form an inflow parameter vector, $\boldsymbol{x}$, which is used as the independent variable. This vector consists of a primary inflow parameter vector, $\boldsymbol{x}_{p}$, and a secondary inflow parameter vector, $\boldsymbol{x}_{s}$. The wind turbine community has a widely held practice of using the horizontal mean wind speed at hub height and a measure of turbulence, such as the standard deviation of horizontal wind speed, as the primary factors that influence fatigue and extreme loads. Accordingly, $\boldsymbol{x}_{\mathrm{p}}$, the primary inflow parameter vector, is defined to consist of the mean $(\mu)$ and standard deviation $(\sigma)$ of horizontal wind speed.

The vector $\boldsymbol{x}_{\mathrm{s}}$ is defined to consist of sixteen secondary inflow wind parameters. Included in this vector are: the vertical wind shear exponent $(\alpha)$, standard deviations of wind speed in the cross-wind and vertical directions $\left(\sigma_{v}\right.$ and $\left.\sigma_{w}\right)$, turbulence kinetic energy $(K E)$, three orthogonal Reynolds stresses ( $u$ 'w', $u$ 'v', and $\left.v^{\prime} w^{\prime}\right)$, local friction velocity $\left(U^{*}\right)$, Obukhov length $(L)$, a stability parameter $(z / L)$, the gradient Richardson number $(R i)$, turbulence length scales in three orthogonal directions $\left(L_{u}, L_{v}\right.$, and $\left.L_{w}\right)$, and the skewness $\left(S_{u}\right)$ and kurtosis $\left(K_{u}\right)$ of horizontal wind speed. Various authors have proposed one ore more of these parameters previously. Rohatgi and Nelson [9] summarize descriptions of many of these parameters. Fragoulis [6], Glinou and Fragoulis [7], Kelley [10], and Kelley and McKenna [11] also propose these and other parameters in studying wind turbine loads. Sutherland $[3,4]$ provides a mathematical description for each of these $\boldsymbol{x}_{\mathrm{s}}$ variables.

Equation (1) summarizes the various primary and secondary inflow parameters used in this study:

$$
\begin{aligned}
\boldsymbol{x}= & \left\{\boldsymbol{x}_{p}, \boldsymbol{x}_{s}\right\} ; \quad \boldsymbol{x}_{p}=\{\mu, \sigma\} ; \\
\boldsymbol{x}_{s}= & \left\{\alpha, \sigma_{v}, \sigma_{w}, K E, u^{\prime} w^{\prime}, u^{\prime} v^{\prime}, v^{\prime} w^{\prime}, \ldots\right. \\
& \left.\ldots U^{*}, L, z / L, R i, L_{u}, L_{v}, L_{w}, S_{u}, K_{u}\right\}
\end{aligned}
$$

\section{$\underline{\text { Regression }}$}

We next describe an initial bivariate linear regression that is performed on the primary inflow parameter vector, $\boldsymbol{x}_{\mathrm{p}}$.

$$
\hat{y}=A_{0} \boldsymbol{x}_{p}+B_{0} ; \quad y-\hat{y}=\varepsilon_{1}
$$

From Eq. (2), the initial residual, $\varepsilon_{1}$, is defined as the difference between the observed value, $y$, of the dependent variable, and the predicted value, $\hat{y}$, resulting from a least squares regression on $\boldsymbol{x}_{\mathrm{p}}$. Variations of the linear model of Eq. (2) were also considered (such as logarithmic models) but they did not improve on the simple linear regression.

After this initial regression is performed, the residual, $\varepsilon_{1}$ is regressed on a single parameter from the secondary inflow parameter vector, $\boldsymbol{x}_{\mathrm{s}}$, as shown in Eq. (3). The selection of one particular element of $\boldsymbol{x}_{\mathrm{s}}$ is made based on the correlation coefficient between $\varepsilon_{1}$ and each element of $\boldsymbol{x}_{\mathrm{s}}$. The element in $\boldsymbol{x}_{\mathrm{s}}$ that has the highest correlation with $\varepsilon_{1}$ is chosen as $x_{s, 1}$.

$\varepsilon_{1}=A_{1} x_{s, 1}+B_{1}+\varepsilon_{2} ; \quad \ldots \quad \varepsilon_{i}=A_{i} x_{s, i}+B_{i}+\varepsilon_{i+1}$
$\theta_{i}=1-S E_{i} / S E_{i-1} ;$

where $\left(\varepsilon_{i}^{2}\right)_{j}$ represents the squared residual from data set $j$ of the $N$ sets (note, $N$ is equal to 491 when all the LIST data sets are used) after the $i$ th regression. Only linear models were considered for regression of the residuals on the secondary inflow parameters.

Equation (3) shows how the residual, $\varepsilon_{i}$, from any step $i$ during this procedure of regression on one element of $\boldsymbol{x}_{\mathrm{s}}$ (indicated here by $x_{s, i}$ ) leads to a new residual, $\varepsilon_{i+1}$ and the regression procedure continues. The degree of influence that any variable, $x_{s, i}$, has on the amplitude of $y$ is indicated by the parameter, $\theta_{i}$, in Eq. (4) since $\theta_{i}$ quantifies the reduction in the sum of the squared residuals in step $i$ (relative to step $i-1$ ). Thus, a small value of $\theta_{i}$ is an indication that there is no significant benefit of the regression on $x_{s, i}$ in influencing or helping explain the response variable, $y$. Alternatively, a large value of $\theta_{i}$, indicates that $x_{s, i}$ does help in predicting or explaining $y$ better. After the initial regression on $x_{s, 1}$, the order of selection of each subsequent element of $\boldsymbol{x}_{\mathrm{s}}$ corresponds to the parameter with the highest correlation coefficient between $\varepsilon_{i}$ and each of the unaccounted for elements of $\boldsymbol{x}_{\mathrm{s}}$. Thus, after each regression step dealing with the parameter, $x_{s, i}$ in the secondary inflow vector, $\boldsymbol{x}_{\mathrm{s}}$, a newly computed correlation matrix of $\varepsilon_{i+1}$ with the remaining parameters of $\boldsymbol{x}_{\mathrm{s}}$ guides the next choice, $x_{s, i+1}$ and the procedure continues; it is stopped when the value of $\theta_{i}$ approaches zero.

This analysis is in sharp contrast to the analyses performed by Fragoulis [6], Glinou and Fragoulis [7], and Sutherland $[1,3]$. In particular, a sequential analysis is presented here, while the other studies use a 
single multivariate regression to determine the influence of all the inflow parameters simultaneously. The current procedure takes into consideration correlation among the secondary inflow parameters while the referenced studies do not.

\section{DATA ANALYSIS}

\section{Dependent Variables}

\section{Fatigue Loads}

The blade root fatigue loads for the flap- and edge-wise bending moment are each considered separately as the dependent variable, $y$. These are characterized using the damage-equivalent fatigue load (see Sutherland $[1,3]$ ) and are determined for two fatigue exponents, $m$, equal to 3 and 10 . The former yields an equivalent fatigue load appropriate for welded steel and the latter for fiberglass composites.

In summary, regression analyses were carried out where $y$ given by Eq. (2) represented four different variables corresponding to damage-equivalent root edge or flap bending moments for $m$ equal to 3 or 10 .

\section{Extreme Loads}

The extreme blade root flap- and edge-wise bending moments are next considered as the dependent variable, $y$. The maximum or extreme load is another measure of the severity of a load spectrum. For this analysis, the single largest maximum flap- and edge-wise bending moment value determined from each ten-minute time series was employed.

In summary, regression analyses were carried out where $y$ given by Eq. (2) represented two different variables corresponding to extreme root edge or flap bending moments.

\section{Numerical Studies}

The regression procedure outlined above and described by Eqs. (1-4) was employed for the analysis of several different response time series.

First, the entire available data set of 491 ten-minute data segments was used to study the dependencies of flap and edge bending moment fatigue (damageequivalent) loads for $m$ equal to 3 and 10 on the various inflow parameters in $\boldsymbol{x}_{p}$ and $\boldsymbol{x}_{s}$. The dependency of flap and edge extreme loads on the inflow parameters was also examined for this data set.
Next, similar analyses were performed on a reduced data set consisting of only those ten-minute data segments with a mean horizontal wind speed greater than the rated wind speed (taken to be $13 \mathrm{~m} / \mathrm{s}$ ). Only sixty-three (63) of the 491 ten-minute records were retained in this set of studies which was carried out to compare and contrast the dependency of each response variable, $y$, on the vector of inflow parameters, $\boldsymbol{x}$, for the entire data set with that for the reduced (aboverated) data sets.

The selection of mean wind speed and standard deviation of wind speed as "primary" inflow parameters was confirmed as being the most appropriate since we found that the correlation coefficient for each of these two parameters with any choice for variable, $y$, was larger than that for any of the secondary inflow parameters in the vector, $\boldsymbol{x}_{s}$.

\section{RESULTS}

\section{The Entire Data Set}

The subset of the LIST data used for this analysis contained 491 ten-minute data records. Each of these records had a mean wind speed that was greater than 7 $\mathrm{m} / \mathrm{s}$.

\section{Fatigue Loads}

Figures 3a-3d show results from the bivariate regression of the equivalent fatigue load portion of $y$ performed on the primary inflow parameter vector, $\boldsymbol{x}_{p}$. Figures $3 \mathrm{a}$ and $3 \mathrm{~b}$ are for the fatigue damage-equivalent edge bending moments for $m$ equal to 3 and 10 , respectively. Similarly, Figs. $3 c$ and $3 d$ are for the damage-equivalent flap bending moments for $m$ equal to 3 and 10, respectively. The regression plane is not shown; instead, the data are shown along with a plot of $y$ versus the mean horizontal wind speed. To represent a range of typical values for the other primary inflow parameter, namely the standard deviation of the horizontal wind speed, $\sigma$ (denoted as sigma in the figures), three lines based on the regression results and representing sigma values of $1.0,1.5$, and $2.0 \mathrm{~m} / \mathrm{s}$ are also shown. These three lines are almost coincident indicating that dependence of the fatigue loads on the standard deviation of the wind speed (sigma) is relatively small for all of these cases.

Table 1 shows correlation coefficients for the residual, $\varepsilon_{1}$ (see Eq. (3)) with elements of the secondary inflow vector, $\boldsymbol{x}_{s}$, after regression of $y$ (in this case, damageequivalent fatigue loads in the edge and flap bending modes for $m$ equal to 3 and 10) on $\boldsymbol{x}_{p}$. The low 
correlation coefficients indicate that performing further regressions on $\boldsymbol{x}_{s}$ will not significantly reduce the residuals compared to what was already achieved after regression of $y$ on $\boldsymbol{x}_{p}$. To illustrate this, Table 2 shows the results of five additional regressions of residuals, $\varepsilon_{i}$, performed on one parameter of $\boldsymbol{x}_{s}$ at a time. Results are shown for all four cases described above (namely, fatigue damage-equivalent edge and flap bending moments with fatigue exponent, $m$ equal to 3 and 10).

As defined earlier, the $\theta_{i}$ term in general describes how much of the variance in residuals is explained by the regression. Note that $\theta_{0}$ is equivalent to the coefficient of determination, $R^{2}$, after the first regression. As an illustration, $\boldsymbol{x}_{p}$ explains anywhere from 17 to 41 percent of the variance of the residuals, or, equivalently, the regression of $y$ on $\boldsymbol{x}_{p}$ never leads to $R^{2}$ values greater than 0.41. The implication is that $y$ cannot be adequately predicted by the primary inflow parameters, $\boldsymbol{x}_{p}$, alone - a fact that can be confirmed by the data shown in Figs. 3a-3d.

Table 2 also shows the sums of squared residuals and the regression coefficients through each step of the procedure. The parameters from $\boldsymbol{x}_{s}$ shown for $i$ equal to 1-5 in the table are selected based on the highest correlation coefficient of that parameter with the residual from the preceding regression. Thus, for example, for the damage-equivalent edge bending moment with $m$ equal to 3 , the parameters of $\boldsymbol{x}_{s}$ selected are in sequence, $\alpha, K E, R_{i}, K_{u}$, and $S_{u}$ (the vertical wind shear exponent, turbulent kinetic energy, gradient Richardson number, and the kurtosis and skewness of the horizontal wind speed). It is clear that when the entire data set is used, none of the parameters in $\boldsymbol{x}_{s}$ has significant influence on reducing the residuals in $y$ in the case of fatigue damage-equivalent bending moments in edge or flap modes. This can be seen by the low values of $\theta_{i}$ for all $i>0$. This also suggests that no secondary inflow parameter usefully contributes to explanation of edge and flap bending fatigue loads once the primary variables of mean wind speed and standard deviation of wind speed are accounted for.

\section{Extreme Loads}

Figures $4 \mathrm{a}$ and $4 \mathrm{~b}$ show results from the bivariate regression on the extreme load portion of $y$ performed on the primary inflow parameter vector, $\boldsymbol{x}_{p}$. Figure $4 \mathrm{a}$ is for the extreme edge bending moment while Fig. $4 \mathrm{~b}$ is for the extreme flap bending moment. Again, the regression plane is not shown; instead, the data are shown along with a plot of $y$ versus the mean horizontal wind speed for three values of the standard deviation of the horizontal wind speed $(1.0,1.5$, and $2.0 \mathrm{~m} / \mathrm{s})$. For extreme loads, the dependence on standard deviation of the wind speed is significantly higher than was the case with the fatigue loads. Hence, the three lines shown in Figs. $4 \mathrm{a}$ and $4 \mathrm{~b}$ are well separated.

Table 3 shows correlation coefficients for the residual, $\varepsilon_{1}$ with elements of the secondary inflow vector, $\boldsymbol{x}_{s}$, after regression of $y$ on $\boldsymbol{x}_{p}$. The correlation coefficients are still fairly low but higher than for the fatigue loads described earlier. Further regressions on $\boldsymbol{x}_{s}$ do not significantly reduce the residuals compared to their values after regression of $y$ on $\boldsymbol{x}_{p}$ as can be seen in Table 4.

In the case of the extreme loads, $\boldsymbol{x}_{p}$ explains almost 70 percent of the variance of the residuals (or, equivalently, the regression of $y$ on $x_{p}$ leads to $R^{2}$ values of nearly 0.7 and much better regression fits of $y$ to $\boldsymbol{x}_{p}$ as may be seen in Figs. $4 \mathrm{a}$ and $4 \mathrm{~b}$ than was the case of the fatigue loads). Table 4 also shows the sums of squared residuals and the regression coefficients through each step of the procedure. Again, as an example, for the extreme flap loads, the parameters of $\boldsymbol{x}_{s}$ selected are in sequence, $L_{w}, S_{u}, K_{u}, \alpha$, and $\sigma_{v}$, but it is clear that when the entire data set is used, none of the parameters in $\boldsymbol{x}_{s}$ has significant influence on reducing the residuals in $y$ in the case of extreme bending moments in edge or flap mode. This can be seen by the low values of $\theta_{i}$ for all $i>0$. This also suggests that no secondary inflow parameter usefully contributes to prediction of edge and flap bending extreme loads.

\section{Reduced Data Set}

The subset of the LIST data used for this analysis contained 63 ten-minute data records. Only records with mean wind speed greater than $13 \mathrm{~m} / \mathrm{s}$ are considered.

The purpose for considering this reduced data set is to determine if the dependencies of fatigue and extreme loads on inflow parameters (primary or secondary) might be different in this above-rated wind speed regime compared to the findings based on the use of the entire data set. Some improvement is expected if the above-rated loads represent a more homogeneous data set.

\section{Fatigue Loads}

Figures 5a-5d show results from the bivariate regression of the equivalent fatigue load portion of $y$ performed on the primary inflow parameter vector, $\boldsymbol{x}_{p}$. Figures $5 \mathrm{a}$ and $5 \mathrm{~b}$ are for the fatigue damage-equivalent 


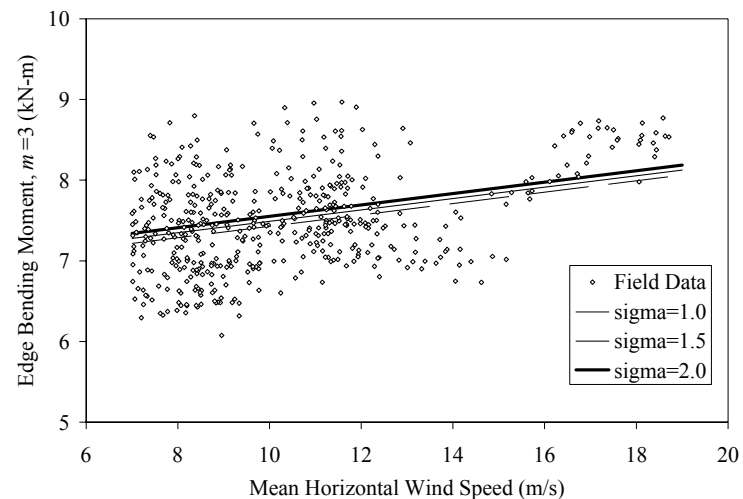

Figure 3a: Equivalent Fatigue Edge BM, $m=3$ versus Wind Speed for the Entire Data Set

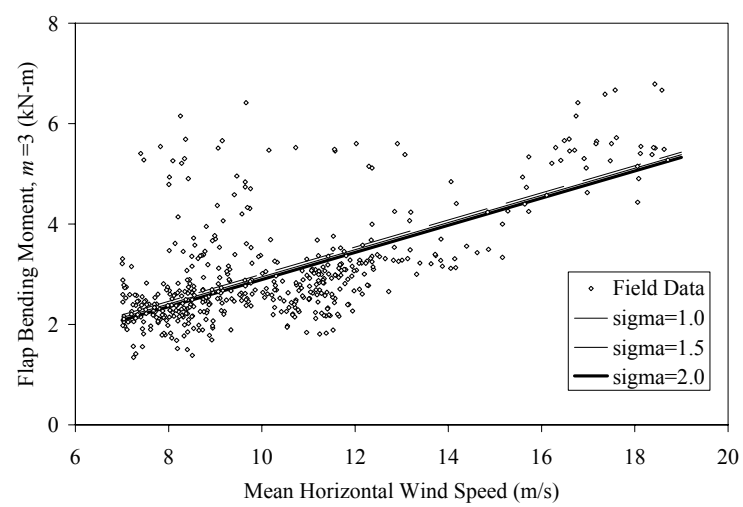

Figure 3c: Equivalent Fatigue Flap BM, $m=3$ versus Wind Speed for the Entire Data Set

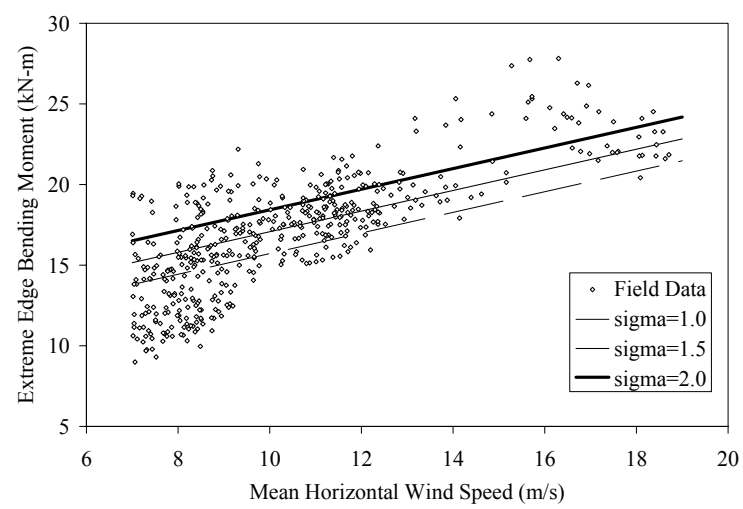

Figure 4a: Extreme Edge BM versus Wind Speed for the Entire Data Set

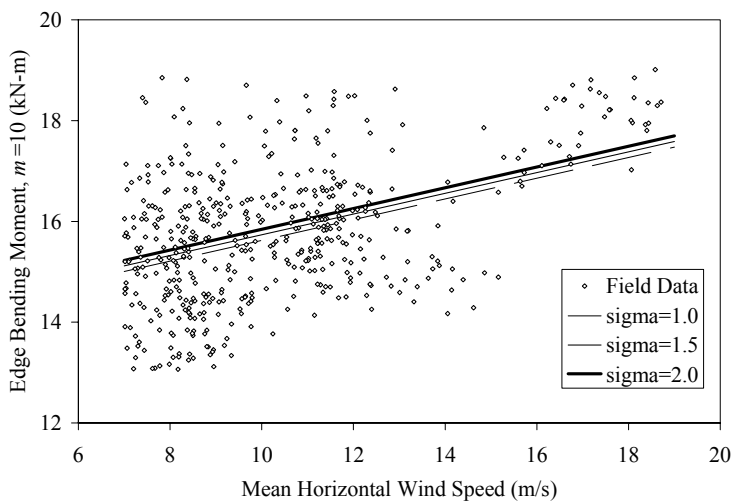

Figure 3b: Equivalent Fatigue Edge BM, $m=10$ versus Wind Speed for the Entire Data Set

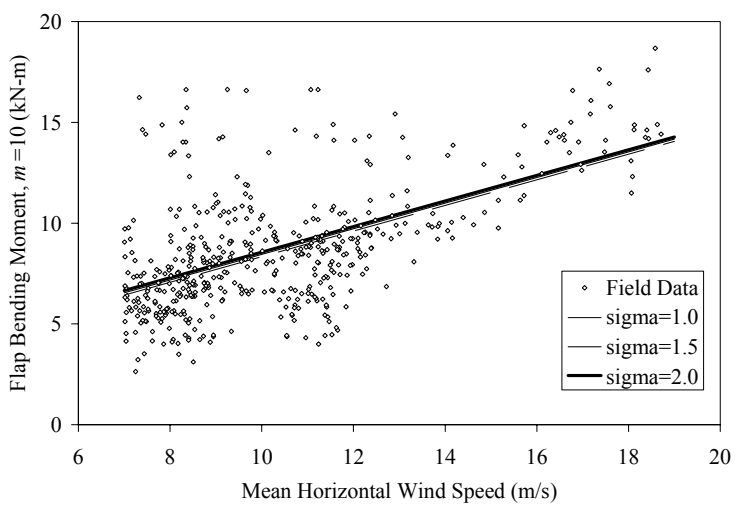

Figure 3d: Equivalent Fatigue Flap BM, $m=10$ versus Wind Speed for the Entire Data Set

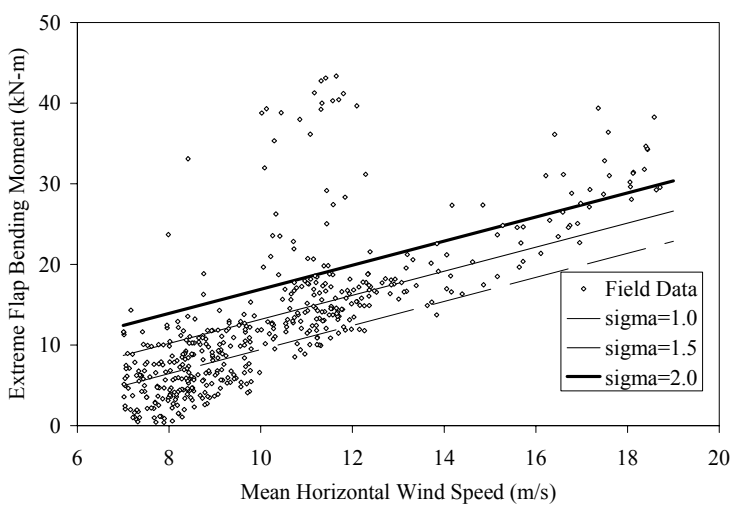

Figure 4b: Extreme Flap BM versus Wind Speed for the Entire Data Set 


\begin{tabular}{|c|c|c|c|c|c|}
\hline & & \multicolumn{2}{|c|}{ Edge } & \multicolumn{2}{c|}{ Flap } \\
\hline$i$ & $x_{s, i}$ & $m=3$ & $m=10$ & $m=3$ & $m=10$ \\
\hline 1 & $\alpha$ & 0.10 & 0.11 & 0.10 & 0.05 \\
\hline 2 & $\sigma_{v}$ & 0.05 & 0.04 & -0.03 & -0.05 \\
\hline 3 & $\sigma_{w}$ & -0.01 & -0.01 & -0.04 & -0.04 \\
\hline 4 & $K E$ & 0.06 & 0.05 & 0.02 & 0.00 \\
\hline 5 & $u^{\prime} w^{\prime}$ & 0.01 & 0.00 & -0.02 & 0.00 \\
\hline 6 & $u^{\prime} v^{\prime}$ & 0.00 & 0.00 & 0.03 & 0.07 \\
\hline 7 & $v^{\prime} w^{\prime}$ & 0.06 & 0.04 & -0.03 & -0.03 \\
\hline 8 & $U^{*}$ & -0.03 & -0.03 & -0.01 & -0.03 \\
\hline 9 & $L$ & -0.02 & -0.01 & 0.02 & 0.02 \\
\hline 10 & $z / L$ & 0.07 & 0.05 & -0.04 & -0.06 \\
\hline 11 & $R i$ & -0.10 & -0.11 & -0.04 & -0.03 \\
\hline 12 & $L_{u}$ & 0.02 & 0.03 & 0.00 & 0.02 \\
\hline 13 & $L_{v}$ & 0.05 & 0.04 & -0.05 & -0.06 \\
\hline 14 & $L_{w}$ & 0.04 & 0.03 & -0.08 & -0.07 \\
\hline 15 & $S_{u}$ & 0.08 & 0.10 & 0.13 & 0.02 \\
\hline 16 & $K_{u}$ & 0.05 & 0.03 & -0.05 & 0.00 \\
\hline
\end{tabular}

Table 1: Correlation Coefficient Matrix for Damage-Equivalent Fatigue Loads using the Entire Data Set.

\begin{tabular}{|c|c|c|c|c|}
\hline$i$ & $x$ & $A_{i}$ & $B_{i}$ & $\theta_{i}$ \\
\hline 0 & $\boldsymbol{x}_{p}$ & {$[0.07,0.12]$} & 6.60 & 0.166 \\
\hline 1 & $\alpha$ & 0.566 & -0.100 & 0.010 \\
\hline 2 & $K E$ & 0.036 & -0.094 & 0.016 \\
\hline 3 & $R i$ & -0.179 & 0.029 & 0.008 \\
\hline 4 & $K_{u}$ & 0.064 & 0.004 & 0.004 \\
\hline 5 & $S_{u}$ & 0.135 & 0.015 & 0.008 \\
\hline
\end{tabular}

(a) Edge Bending Moment, $m=3$.

\begin{tabular}{|c|c|c|c|c|}
\hline$i$ & $x$ & $A_{i}$ & $B_{i}$ & $\theta_{i}$ \\
\hline 0 & $\boldsymbol{x}_{p}$ & {$[0.21,0.22]$} & 13.35 & 0.232 \\
\hline 1 & $\alpha$ & 1.327 & -0.235 & 0.012 \\
\hline 2 & $K E$ & 0.076 & -0.196 & 0.015 \\
\hline 3 & $R i$ & -0.395 & 0.064 & 0.009 \\
\hline 4 & $S_{u}$ & 0.270 & 0.031 & 0.006 \\
\hline 5 & $K_{u}$ & 0.153 & 0.010 & 0.005 \\
\hline
\end{tabular}

(b) Edge Bending Moment, $m=10$.

\begin{tabular}{|c|c|c|c|c|}
\hline$i$ & $x$ & $A_{i}$ & $B_{i}$ & $\theta_{i}$ \\
\hline 0 & $\boldsymbol{x}_{p}$ & {$[0.27,-0.10]$} & 0.40 & 0.409 \\
\hline 1 & $S_{u}$ & 0.317 & 0.036 & 0.017 \\
\hline 2 & $L_{w}$ & -0.007 & 0.111 & 0.008 \\
\hline 3 & $K E$ & 0.033 & -0.085 & 0.006 \\
\hline 4 & $\alpha$ & 0.652 & -0.115 & 0.006 \\
\hline 5 & $L_{u}$ & 0.001 & -0.068 & 0.003 \\
\hline
\end{tabular}

(c) Flap Bending Moment, $m=3$.

\begin{tabular}{|c|c|c|c|c|}
\hline$i$ & $x$ & $A_{i}$ & $B_{i}$ & $\theta_{i}$ \\
\hline 0 & $\boldsymbol{x}_{p}$ & {$[0.64,0.23]$} & 1.75 & 0.356 \\
\hline 1 & $L_{w}$ & -0.015 & 0.244 & 0.005 \\
\hline 2 & $u^{\prime} v^{\prime}$ & 0.234 & 0.003 & 0.004 \\
\hline 3 & $z / L$ & -0.009 & 0.006 & 0.003 \\
\hline 4 & $L_{u}$ & 0.003 & -0.194 & 0.003 \\
\hline 5 & $L_{v}$ & -0.002 & 0.115 & 0.002 \\
\hline
\end{tabular}

(d) Flap Bending Moment, $m=10$.

Table 2: Regression Results for Damage-Equivalent Fatigue Loads using the Entire Data Set. 


\begin{tabular}{|c|c|c|c|}
\hline$i$ & $x_{s, i}$ & Edge & Flap \\
\hline 1 & $\alpha$ & -0.14 & -0.01 \\
\hline 2 & $\sigma_{v}$ & 0.11 & 0.15 \\
\hline 3 & $\sigma_{w}$ & 0.10 & 0.04 \\
\hline 4 & $K E$ & 0.00 & 0.09 \\
\hline 5 & $u^{\prime} w^{\prime}$ & -0.02 & -0.04 \\
\hline 6 & $u^{\prime} v^{\prime}$ & 0.03 & -0.01 \\
\hline 7 & $v^{\prime} w^{\prime}$ & -0.24 & -0.01 \\
\hline 8 & $U^{*}$ & 0.06 & 0.05 \\
\hline 9 & $L$ & 0.02 & 0.01 \\
\hline 10 & $z / L$ & -0.06 & 0.05 \\
\hline 11 & $R i$ & 0.14 & 0.01 \\
\hline 12 & $L_{u}$ & 0.09 & 0.03 \\
\hline 13 & $L_{v}$ & 0.07 & 0.14 \\
\hline 14 & $L_{w}$ & 0.09 & 0.17 \\
\hline 15 & $S_{u}$ & 0.16 & 0.14 \\
\hline 16 & $K_{u}$ & 0.03 & 0.02 \\
\hline
\end{tabular}

Table 3: Correlation Coefficient Matrix for Extreme Loads using the Entire Data Set.

flap bending moments for $m$ equal to 3 and 10 , respectively. The data are shown along with a plot of $y$ versus the mean horizontal wind speed. To represent a range of typical values for the other primary inflow parameter, namely the standard deviation of the horizontal wind speed (sigma), three lines based on the bivariate regression results and representing sigma values of $1.0,1.5$, and $2.0 \mathrm{~m} / \mathrm{s}$ are also shown. The gradient of bending moment with respect to standard deviation of the wind speed is greater than when the entire data set was used.

Table 5 shows correlation coefficients for the residual, $\varepsilon_{1}$ with elements of the secondary inflow vector, $\boldsymbol{x}_{s}$, after regression of $y$ (in this case, damage-equivalent fatigue loads in the edge and flap bending modes for $m$ equal to 3 and 10) on $\boldsymbol{x}_{p}$. The correlation coefficients are higher now when the reduced data set is used as compared to those with the entire data set (see Table 1). This indicates that performing further regressions on $\boldsymbol{x}_{s}$ might help to reduce the residuals further after the regression of $y$ on $x_{p}$. To illustrate this, Table 6 shows the results of five additional regressions of residuals, $\varepsilon_{i}$, performed on one parameter of $\boldsymbol{x}_{s}$ at a time. Results are shown for all four cases described above (namely, fatigue damage-equivalent edge and flap bending moments with fatigue exponent, $m$ equal to 3 and 10).

\begin{tabular}{|c|c|c|c|c|}
\hline$i$ & $x$ & $A_{i}$ & $B_{i}$ & $\theta_{i}$ \\
\hline 0 & $\boldsymbol{x}_{p}$ & {$[0.64,2.72]$} & 6.60 & 0.695 \\
\hline 1 & $v^{\prime} w^{\prime}$ & -4.28 & 0.00 & 0.060 \\
\hline 2 & $S_{u}$ & 0.82 & 0.09 & 0.021 \\
\hline 3 & $\alpha$ & -3.27 & 0.58 & 0.028 \\
\hline 4 & $K E$ & -0.10 & 0.26 & 0.010 \\
\hline 5 & $R i$ & 0.58 & -0.09 & 0.007 \\
\hline
\end{tabular}

(a) Extreme Edge Bending Moment.

\begin{tabular}{|c|c|c|c|c|}
\hline$i$ & $x$ & $A_{i}$ & $B_{i}$ & $\theta_{i}$ \\
\hline 0 & $\boldsymbol{x}_{p}$ & {$[1.49,7.47]$} & -12.94 & 0.683 \\
\hline 1 & $L_{w}$ & 0.08 & -1.30 & 0.029 \\
\hline 2 & $S_{u}$ & 1.96 & 0.22 & 0.017 \\
\hline 3 & $K_{u}$ & 0.98 & 0.07 & 0.011 \\
\hline 4 & $\alpha$ & 3.38 & -0.60 & 0.004 \\
\hline 5 & $\sigma_{v}$ & 0.85 & -1.02 & 0.009 \\
\hline
\end{tabular}

(b) Extreme Flap Bending Moment.

\section{Table 4: Regression Results for Extreme Loads using the Entire Data Set.}

With this reduced data set, $\boldsymbol{x}_{p}$ explains anywhere from 66 to 79 percent of the variance of the residuals, or, equivalently, the regression of $y$ on $\boldsymbol{x}_{p}$ leads to $R^{2}$ values greater than 0.65 . In all cases, $y$ is predicted, to a moderate degree, by the primary inflow parameters, $\boldsymbol{x}_{p}$, alone - this can be confirmed by the reasonable fits to the data as seen in Figs. 5a-5d.

For purposes of comparison with the analysis considering the entire data set, for the damageequivalent edge bending moment with $m$ equal to 3, the parameters of $\boldsymbol{x}_{s}$ selected are in sequence, $\alpha, \sigma_{v}, U^{*}, z / L$, and $L$. It is clear that when the reduced data set is used, at least one or two parameters in $\boldsymbol{x}_{s}$ have some influence on reducing the residuals in $y$ in the case of fatigue damage-equivalent bending moments in edge or flap mode. This can be seen by values of $\theta_{i}$ greater than 0.10 in a few cases (e.g., with the wind shear exponent, $\alpha$, in the case of edge-wise fatigue loads). Thus, in a few cases, one or more secondary inflow parameters, in addition to the primary inflow parameters, might contribute to prediction of edge and flap bending fatigue loads, at least relative to the case where the entire data set was used.

In summary, both primary and secondary inflow parameters help to a greater extent in describing fatigue loads when the reduced data set that includes only the above-rated wind speed data is used. 


\begin{tabular}{|c|c|c|c|c|c|}
\hline & & \multicolumn{2}{|c|}{ Edge } & \multicolumn{2}{c|}{ Flap } \\
\hline$i$ & $x_{s, i}$ & $m=3$ & $m=10$ & $m=3$ & $m=10$ \\
\hline 1 & $\alpha$ & 0.32 & 0.27 & 0.20 & 0.16 \\
\hline 2 & $\sigma_{v}$ & 0.28 & 0.29 & 0.30 & 0.31 \\
\hline 3 & $\sigma_{w}$ & 0.23 & 0.25 & 0.11 & 0.12 \\
\hline 4 & $K E$ & 0.19 & 0.20 & 0.18 & 0.20 \\
\hline 5 & $u^{\prime} w^{\prime}$ & 0.08 & 0.06 & 0.13 & 0.13 \\
\hline 6 & $u^{\prime} v^{\prime}$ & 0.04 & 0.10 & 0.28 & 0.30 \\
\hline 7 & $v^{\prime} w^{\prime}$ & 0.14 & 0.10 & 0.03 & -0.07 \\
\hline 8 & $U^{*}$ & -0.08 & -0.05 & -0.12 & -0.13 \\
\hline 9 & $L$ & 0.12 & 0.11 & 0.21 & 0.24 \\
\hline 10 & $z / L$ & 0.13 & 0.12 & -0.04 & -0.14 \\
\hline 11 & $R i$ & -0.14 & -0.11 & -0.04 & 0.03 \\
\hline 12 & $L_{u}$ & -0.05 & -0.03 & -0.05 & -0.01 \\
\hline 13 & $L_{v}$ & 0.05 & 0.06 & 0.07 & 0.17 \\
\hline 14 & $L_{w}$ & -0.18 & -0.14 & -0.14 & -0.06 \\
\hline 15 & $S_{u}$ & 0.21 & 0.21 & 0.09 & 0.10 \\
\hline 16 & $K_{u}$ & -0.04 & -0.02 & -0.07 & 0.06 \\
\hline
\end{tabular}

Table 5: Correlation Coefficient Matrix for Damage-Equivalent Fatigue Loads using the Reduced Data Set.

\section{Extreme Loads}

Figures $6 \mathrm{a}$ and $6 \mathrm{~b}$ show results from the bivariate regression of the extreme load portion of $y$ performed on the primary inflow parameter vector, $\boldsymbol{x}_{p}$. Figure 6a is for the extreme edge bending moment while Fig. $6 \mathrm{~b}$ is for the extreme flap bending moment. The data are shown along with a plot of $y$ versus the mean horizontal wind speed for three values of the standard deviation of the horizontal wind speed $(1.0,1.5$, and $2.0 \mathrm{~m} / \mathrm{s})$. Extreme edge loads show little dependence on mean wind speed for this reduced data set but gradients with respect to standard deviation of wind speed for both edge and flap extreme loads are higher for this reduced data set as may be confirmed from Figs. 6a and $6 \mathrm{~b}$ compared to results shown in Figs. $4 a$ and $4 b$ for the entire data set.

Table 7 shows correlation coefficients for the residual, $\varepsilon_{1}$ with elements of the secondary inflow vector, $\boldsymbol{x}_{s}$, after regression of $y$ (in this case, extreme loads in the edge and flap bending modes) on $\boldsymbol{x}_{p}$. The correlation coefficients are somewhat higher now when the reduced data set is used as compared to those with the entire data set (see Table 3). They are, however, lower correlation coefficients than were obtained for fatigue loads with this reduced data set (see Table 5). Thus, further regressions on $\boldsymbol{x}_{s}$ do not significantly reduce the residuals compared to their values after regression of $y$ on $\boldsymbol{x}_{p}$ as can be seen in Table 8 .

In the case of the extreme loads, $\boldsymbol{x}_{p}$ explains as much as 80 percent of the variance of the residuals for the flap bending moment extreme but only 31 percent for the edge bending moment extreme. This is in contrast to the results when the entire data set was used when for both, flap and edge extremes; $R^{2}$ values of about 70 percent were computed. Table 8 also shows the sums of squared residuals and the regression coefficients through each step of the procedure. Again, it is clear that when the reduced data set is used, none of the parameters in $\boldsymbol{x}_{s}$ has significant influence on reducing the residuals in $y$ in the case of extreme bending moments in edge or flap mode. This can be seen by the low values of $\theta_{i}$ for all $i>0$. This also suggests that no secondary inflow parameter usefully contributes to prediction of edge and flap bending extreme loads after the primary variables of mean wind speed and standard deviation of wind speed are accounted for.

\section{DISCUSSION OF CORRELATION}

The degree of correlation among the various primary and secondary inflow parameters makes it difficult to unambiguously identify the most important parameters that influence turbine loads. When one parameter is strongly correlated with another, even though each might be influential to some extent, the first might serve as a surrogate for the second and the importance of the second might be minimized and vice-versa. In this study, after accounting for the first parameter, the influence of the second will have diminished in part due to the strong correlation with the first. Here, we have taken a first step into dealing with issues of correlation by assuming that the mean wind speed and the standard deviation of wind speed will be treated as the "primary" variables of interest. This assumption is based both on design practice and on the physical insight that steady winds in combination with the gross amount of roughness are reasonable first considerations in the design of wind turbines.

The data set under study suggests that there exists strong correlation between, on the one hand, any one of the two primary inflow parameters, and, on the other hand, one or more of the sixteen secondary inflow parameters. As seen in Table 9, only about five of the sixteen secondary parameters are relatively uncorrelated with the primary parameters of mean wind speed and standard deviation of wind speed. Therefore, each of the other eleven parameters has their relationship with or influence on the response variables 


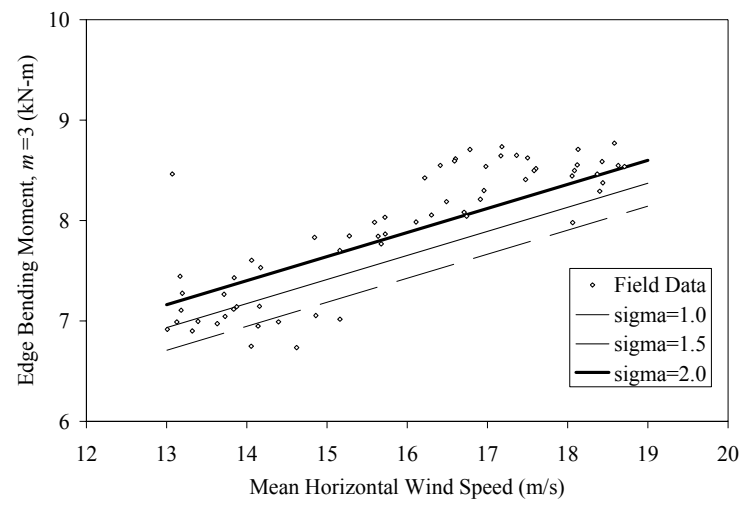

Figure 5a: Equivalent Fatigue Edge BM, $m=3$ versus Wind Speed for the Reduced Data Set

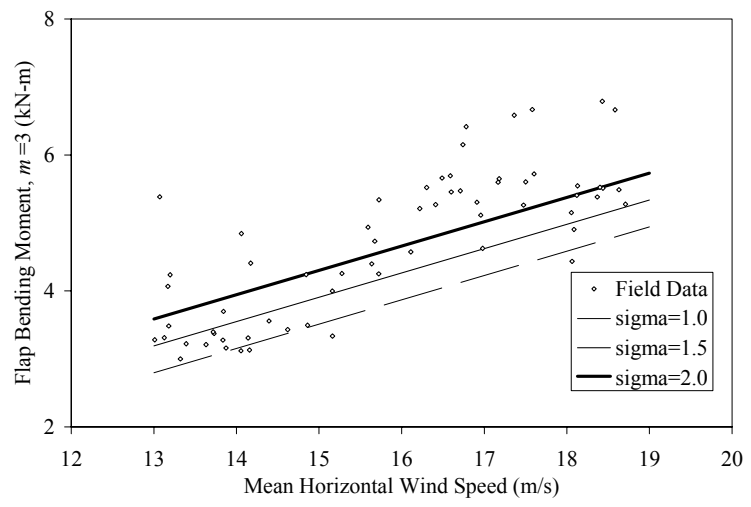

Figure 5c: Equivalent Fatigue Flap BM, $m=3$ versus Wind Speed for the Reduced Data Set

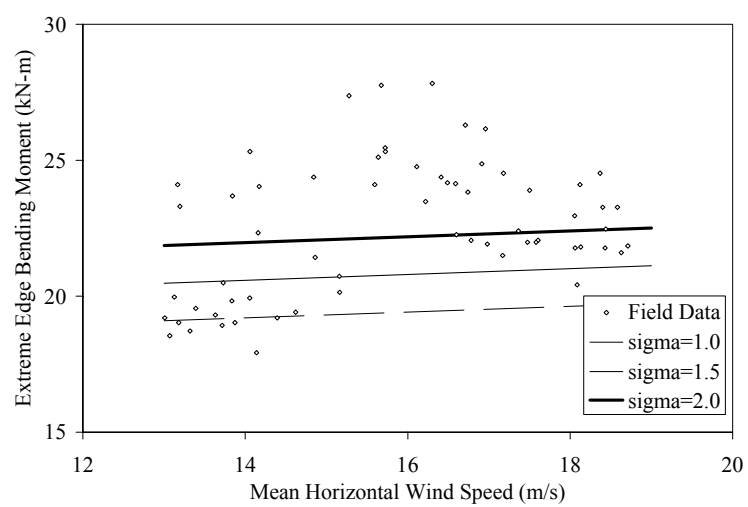

Figure 6a: Extreme Edge BM versus Wind Speed for the Reduced Data Set

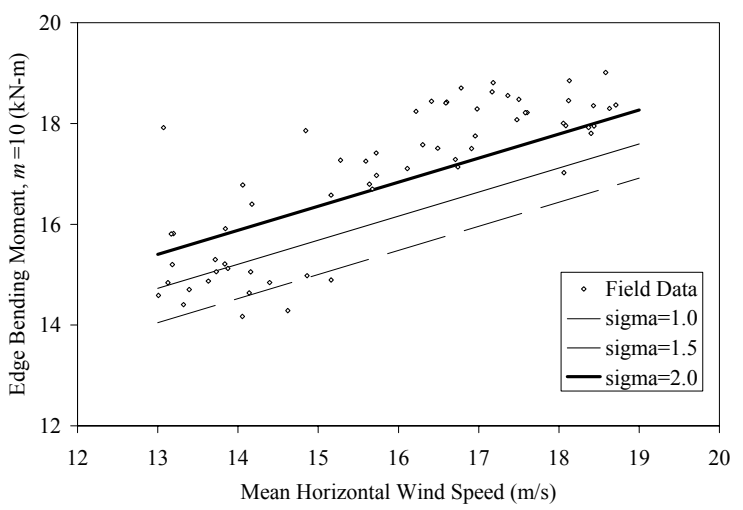

Figure 5b: Equivalent Fatigue Edge BM, $m=10$ versus Wind Speed for the Reduced Data Set

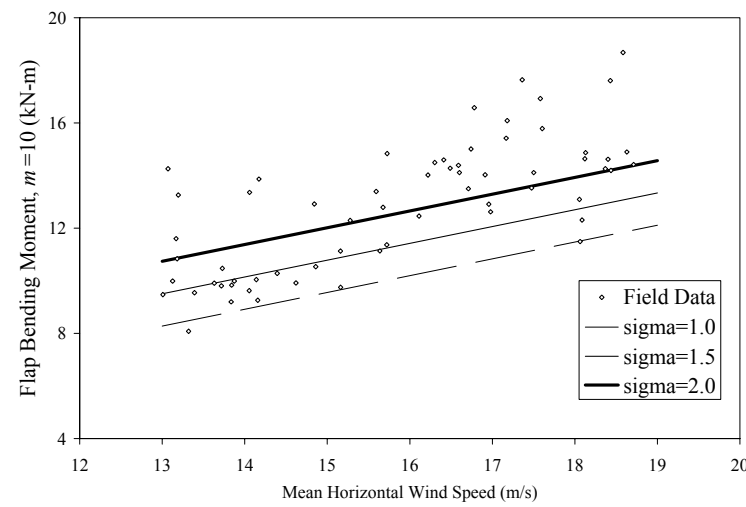

Figure 5d: Equivalent Fatigue Flap BM, $m=10$ versus Wind Speed for the Reduced Data Set

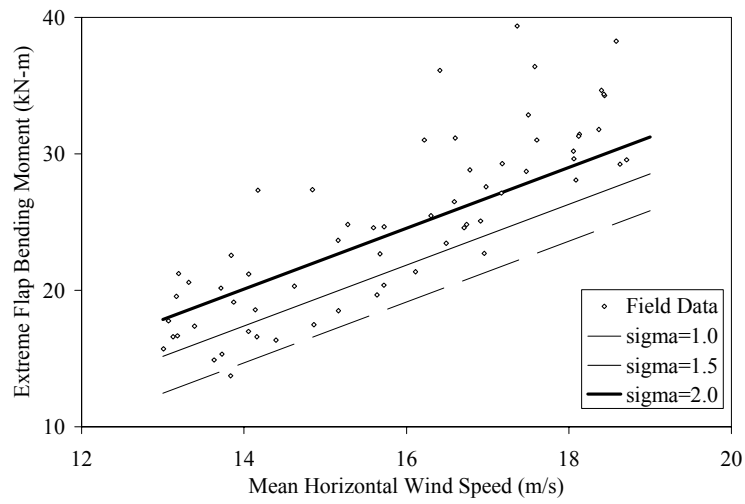

Figure 6b: Extreme Flap BM versus Wind Speed for the Reduced Data Set 


\begin{tabular}{|c|c|c|c|c|}
\hline$i$ & $x$ & $A_{i}$ & $B_{i}$ & $\theta_{i}$ \\
\hline 0 & $\boldsymbol{x}_{p}$ & {$[0.24,0.46]$} & 3.14 & 0.784 \\
\hline 1 & $\alpha$ & 5.11 & -0.68 & 0.100 \\
\hline 2 & $\sigma_{v}$ & 0.20 & -0.38 & 0.123 \\
\hline 3 & $U^{*}$ & -0.50 & 0.44 & 0.080 \\
\hline 4 & $z / L$ & 0.49 & 0.05 & 0.031 \\
\hline 5 & $L$ & 0.00 & 0.02 & 0.043 \\
\hline
\end{tabular}

(a) Edge Bending Moment, $m=3$.

\begin{tabular}{|c|c|c|c|c|}
\hline$i$ & $x$ & $A_{i}$ & $B_{i}$ & $\theta_{i}$ \\
\hline 0 & $\boldsymbol{x}_{p}$ & {$[0.48,1.36]$} & 6.48 & 0.790 \\
\hline 1 & $\sigma_{v}$ & 0.39 & -0.76 & 0.087 \\
\hline 2 & $\alpha$ & 11.67 & -1.56 & 0.114 \\
\hline 3 & $U^{*}$ & -0.97 & 0.85 & 0.059 \\
\hline 4 & $L$ & 0.00 & 0.03 & 0.025 \\
\hline 5 & $z / L$ & 1.10 & 0.11 & 0.030 \\
\hline
\end{tabular}

(b) Edge Bending Moment, $m=10$.

\begin{tabular}{|c|c|c|c|c|}
\hline$i$ & $x$ & $A_{i}$ & $B_{i}$ & $\theta_{i}$ \\
\hline 0 & $\boldsymbol{x}_{p}$ & {$[0.36,0.79]$} & -2.64 & 0.707 \\
\hline 1 & $\sigma_{v}$ & 0.34 & -0.66 & 0.091 \\
\hline 2 & $u^{\prime} v^{\prime}$ & 0.21 & 0.00 & 0.083 \\
\hline 3 & $u^{\prime} w^{\prime}$ & 0.51 & 0.40 & 0.064 \\
\hline 4 & $\alpha$ & 8.13 & -1.09 & 0.088 \\
\hline 5 & $L$ & 0.00 & 0.05 & 0.078 \\
\hline
\end{tabular}

(c) Flap Bending Moment, $m=3$.

\begin{tabular}{|c|c|c|c|c|}
\hline$i$ & $x$ & $A_{i}$ & $B_{i}$ & $\theta_{i}$ \\
\hline 0 & $\boldsymbol{x}_{p}$ & {$[0.64,2.46]$} & -2.47 & 0.669 \\
\hline 1 & $\sigma_{v}$ & 0.87 & -1.67 & 0.098 \\
\hline 2 & $u^{\prime} v^{\prime}$ & 0.55 & 0.00 & 0.099 \\
\hline 3 & $U^{*}$ & -2.20 & 1.92 & 0.070 \\
\hline 4 & $\alpha$ & 16.85 & -2.26 & 0.067 \\
\hline 5 & $L$ & 0.00 & 0.12 & 0.083 \\
\hline
\end{tabular}

(d) Flap Bending Moment, $m=10$.

Table 6: Regression Results for Damage-Equivalent Fatigue Loads using the Reduced Data Set.

\begin{tabular}{|c|c|c|c|}
\hline$i$ & $x_{s, i}$ & Edge & Flap \\
\hline 1 & $\alpha$ & -0.17 & -0.01 \\
\hline 2 & $\sigma_{v}$ & 0.18 & 0.26 \\
\hline 3 & $\sigma_{w}$ & 0.03 & 0.11 \\
\hline 4 & $K E$ & 0.09 & 0.17 \\
\hline 5 & $u^{\prime} w^{\prime}$ & 0.06 & 0.16 \\
\hline 6 & $u^{\prime} v^{\prime}$ & 0.03 & 0.20 \\
\hline 7 & $v^{\prime} w^{\prime}$ & -0.02 & 0.09 \\
\hline 8 & $U^{*}$ & -0.05 & -0.15 \\
\hline 9 & $L$ & 0.07 & 0.08 \\
\hline 10 & $z / L$ & -0.03 & -0.16 \\
\hline 11 & $R i$ & 0.07 & 0.21 \\
\hline 12 & $L_{u}$ & 0.11 & -0.08 \\
\hline 13 & $L_{v}$ & 0.00 & 0.01 \\
\hline 14 & $L_{w}$ & -0.04 & 0.16 \\
\hline 15 & $S_{u}$ & 0.07 & 0.16 \\
\hline 16 & $K_{u}$ & 0.11 & -0.01 \\
\hline
\end{tabular}

Table 7: Correlation Coefficient Matrix for Extreme Loads using the Reduced Data Set.

\begin{tabular}{|c|c|c|c|c|}
\hline$i$ & $x$ & $A_{i}$ & $B_{i}$ & $\theta_{i}$ \\
\hline 0 & $\boldsymbol{x}_{p}$ & {$[0.11,2.77]$} & -21.95 & 0.313 \\
\hline 1 & $\sigma_{v}$ & 0.72 & -1.39 & 0.032 \\
\hline 2 & $\alpha$ & -15.48 & 2.07 & 0.021 \\
\hline 3 & $L_{v}$ & 0.00 & 0.48 & 0.019 \\
\hline 4 & $K_{u}$ & 0.79 & 0.21 & 0.019 \\
\hline 5 & $u^{\prime} v^{\prime}$ & 0.15 & 0.00 & 0.003 \\
\hline
\end{tabular}

(a) Extreme Edge Bending Moment.

\begin{tabular}{|c|c|c|c|c|}
\hline$i$ & $x$ & $A_{i}$ & $B_{i}$ & $\theta_{i}$ \\
\hline 0 & $\boldsymbol{x}_{p}$ & {$[2.23,5.41]$} & 14.94 & 0.797 \\
\hline 1 & $\sigma_{v}$ & 1.49 & -2.87 & 0.066 \\
\hline 2 & $u^{\prime} w^{\prime}$ & 2.64 & 2.08 & 0.060 \\
\hline 3 & $u^{\prime} v^{\prime}$ & 0.76 & -0.01 & 0.044 \\
\hline 4 & $L_{w}$ & 0.06 & -1.44 & 0.039 \\
\hline 5 & $L_{u}$ & -0.01 & 1.51 & 0.039 \\
\hline
\end{tabular}

(b) Extreme Flap Bending Moment.

Table 8: Regression Results for Extreme Loads using the Reduced Data Set.

$$
\begin{array}{|c|c|c|c|c|c|c|c|c|c|c|c|c|c|c|c|c|}
\hline & \alpha & \sigma_{v} & \sigma_{w} & K E & u^{\prime} w^{\prime} & u^{\prime} v^{\prime} & v^{\prime} w^{\prime} & U^{*} & L & z / L & R i & L_{u} & L_{v} & L_{w} & S_{u} & K_{u} \\
\hline \mu & 0.33 & 0.67 & 0.73 & 0.64 & 0.68 & 0.02 & 0.03 & 0.68 & 0.10 & 0.02 & 0.09 & 0.48 & 0.52 & 0.40 & 0.05 & 0.14 \\
\hline \sigma & 0.71 & 0.79 & 0.87 & 0.94 & 0.75 & 0.03 & 0.03 & 0.74 & 0.09 & 0.07 & 0.45 & 0.64 & 0.56 & 0.57 & 0.05 & 0.20 \\
\hline
\end{array}
$$

Table 9: Correlation Coefficients between the Sixteen Secondary Parameters and the Primary Parameters of Wind Speed Mean $(\mu)$ and Standard Deviation $(\sigma)$. 
largely accounted for by the relationship between the response and the mean and/or standard deviation of the wind speed. That they contribute so little additional explanation (at most $12 \%$ in one particular case and usually much less) implies that there is very little evidence to suggest that they should replace the mean and standard deviation as descriptive parameters. Nevertheless, the fact that one parameter can act as a surrogate for another leaves open the possibility that some secondary parameter may in fact be important in driving the magnitude of turbine response.

\section{CONCLUSIONS}

Data available on inflow and structural response from the LIST program have provided an opportunity to study the influence of various inflow parameters on fatigue and extreme wind turbine loads. A procedure is presented that employed several steps involving regression of loads on primary and secondary inflow parameters. Residuals obtained in each regression step helped in identifying which inflow parameter might be a candidate for regression after more important parameters had already been considered. A systematic approach was followed for damage-equivalent fatigue loads with fatigue exponents of 3 and 10 , and for extreme loads representing root flap and edge bending moments.

A reduced data set, including only those ten-minute records with a mean wind speed above the rated wind speed $(13 \mathrm{~m} / \mathrm{s})$, was employed in an analysis to compare operation above rated (i.e., stalled blades) with the entire data set.

In general, it was found that when the entire data set was used, the fatigue and extreme loads showed few dependencies on either the primary or the secondary inflow parameters. For extreme loads, the dependencies were more evident than for fatigue, especially with the primary inflow parameters.

When the reduced data set was employed, the dependencies of loads on primary inflow parameters were found to be greater than with the entire data set including wind speeds lower than rated as well. Regression fits on the primary inflow parameters were markedly improved. A few secondary inflow parameters related to wind shear $(\alpha)$ and crosswind variability $\left(\sigma_{\mathrm{v}}\right)$ were identified as somewhat important, especially for fatigue loads.
Results from this study suggest that the large correlation coefficients between several of the secondary parameters individually and each of the primary parameters make it difficult for the secondary parameters to provide any additional explanation of turbine response once the primary parameters have been accounted for.

\section{ACKNOWLEDGEMENTS}

The authors gratefully acknowledge the financial support provided by Grant No. 003658-0272-2001 awarded through the Advanced Research Program of the Texas Higher Education Coordinating Board.

\section{REFERENCES}

1. Sutherland, H.J., P.L. Jones, and B. Neal, 2001, "The Long-Term Inflow and Structural Test Program," 2001 ASME Wind Energy Symposium, AIAA-2001-0039, pp. 1-12.

2. Berg, D., and J. Zayas, 2001, Accurate Time-Linked Data Acquisition System Field Deployment and Operational Experience, 2001 ASME Wind Energy Symposium, AIAA/ASME, pp. 153-161.

3. Sutherland, H.J., 2002, "Analysis of the Structural and Inflow Data from the LIST Turbine," JSEE, in publication.

4. Sutherland, H.J., 2001, "Preliminary Analysis of the Structural and Inflow Data from the LIST Turbine," 2001 ASME Wind Energy Symposium, AIAA-20010041, pp. 1-12.

5. Sutherland, H.J., 1999, On the Fatigue Analysis of Wind Turbines, SAND99-0089, Sandia National Laboratories, Albuquerque, NM, $132 \mathrm{p}$.

6. Fragoulis, A.N., 1997, "The Complex Terrain Wind Environment and Its Effects on the Power Output and Loading of Wind Turbines," 1997 ASME Wind Energy Symposium, AIAA/ASME, pp. 33-40.

7. G. Glinou and A. Fragoulis, eds., 1996, Mounturb Final Report, 3 vols., JOU2-CT93-0378.

8. Kelley, N.D., "The Identification of Inflow Fluid Dynamics Parameters that can be Used to Scale Fatigue Loading Spectra of Wind Turbine Structural Components," 1994 ASME Wind Energy Symposium, ASME, pp. 181-196. 
9. Rohatgi, J.S., and V. Nelson, 1994, Wind Characteristics, An Analysis for the Generation of Wind Power, Alternative Energy Institute, Canyon, TX, 237p.

10. Kelley, N.D., and H.E. McKenna, 1996, "The Evaluation of a Turbulent Loads Characterization System." 1996 ASME Wind Energy Symposium,
ASME, pp. 69-77.

11. Kelley, N.D., A.D. Wright, M.L. Buhl, Jr., and J.L. Tangler, 1997, "Long-Term Simulation of Turbulence-Induced Loads Using the SNL WINd3D, FAST, YawDyn, and ADAMS Numerical Codes," 1997 ASME Wind Energy Symposium, ASME, pp. 74-85. 